\title{
Clinical Outcomes and Prognostic Factors in HER-2 Positive Breast Cancer with Brain Metastasis: A Single-centre Experience
}

\author{
Serdar Karakaya, Ibrahim Karadag, Ozturk Ates, Omur Berna Cakmak Oksuzoglu and Umut Demirci \\ Department of Medical Oncology, Dr. Abdurrahman Yurtaslan Ankara Oncology Training and Research Hospital, Ankara, Turkey
}

\begin{abstract}
Objective: To determine the characteristics and prognosis of brain metastasised HER-2 positive breast cancer (BC) patients. Study Design: Descriptive study.

Place and Duration of Study: Ankara Oncology Training and Research Hospital, Turkey between 2000 and 2019.

Methodology: HER-2 positive BC patients were analysed retrospectively and 105 patients were included in the study. Age 18 years and over, HER-2 positive BC, with BM (brain metastases) were the inclusion criteria. Patients with secondary malignancies, those with missing data, and irregular follow-up were excluded from the study. The age, type of treatment, Eastern Cooperative Oncology Group Performance Status (ECOG PS) score, BM date, and the last contact date of the patients were obtained from the hospital records. The Kaplan-Meier method was used to determine the time to BM and OS. Independent factors affecting $\mathrm{OS}$ and time to BM were determined using the Cox regression model.

Results: Patients with ECOG PS score of 0-1 at the time of the BM had 19 months median overall survival (OS), while patients with ECOG PS score of 2 had 8 months $(p<0.01)$. Median OS after BM was 32 and 14 months for patients with one BM and patients with multiple BM, respectively $(p<0.01)$. Multivariate cox regression analyses revealed that time to progression of BM was shorter in patients with high-grade tumors compared to patients with low-grade tumors $(p=0.048)$, and in patients with denovo metastasis compared to patients without de-novo metastasis $(p=0.003)$.

Conclusion: Tumor grade and de-novo metastasis (extracranial metastasis at the time of diagnosis) are independent predictive factors that may cause the earlier occurrence of BM and affect mortality in BC patients.
\end{abstract}

Key Words: Brain metastasis, Breast cancer, HER-2 positive, Metastasis.

How to cite this article: Karakaya S, Karadag I, Ates O, Oksuzoglu OBC, Demirci U. Clinical Outcomes and Prognostic Factors in HER-2 Positive Breast Cancer with Brain Metastasis: A Single-centre Experience. J Coll Physicians Surg Pak 2021; 31(02):166-170.

\section{INTRODUCTION}

Breast cancer $(\mathrm{BC})$ is the most frequently diagnosed cancer in womenglobally and it is a genetically and pathologically heterogeneous disease. ${ }^{1}$ Pathologically, BC is categorised into four subgroups, according to estrogen receptor, progesterone receptor, and human epidermal growth factor receptor 2 (HER-2) basis. $^{2}$ The metastasis potential of different BC subgroups to different locations differs. BC can metastasise into bone, lungs, liver and brain. Brain metastasis (BM) is commonly seen in triple negative (25-75\%) and HER-2 positive subgroups (11-20\%); whereas, it is seen in $8-15 \%$ luminal BC patients. ${ }^{3,4} \mathrm{BM}$ is common during the course of HER-2 positive metastatic $B C{ }^{3,5-9}$ This leads to poorsurvival and worse quality of life. ${ }^{10}$

Correspondence to: Dr. Ibrahim Karadag, Department of Medical Oncology, Dr. Abdurrahman Yurtaslan Ankara Oncology Training and Research Hospital, Ankara, Turkey E-mail: ikaradag58@gmail.com

Received: October 22, 2020; Revised: December 31, 2020; Accepted: February 03, 2021

DOI: https://doi.org/10.29271/jcpsp.2021.02.166
In recent years, the life expectancy of $\mathrm{BC}$ patients with $\mathrm{BM}$ increased with the use of HER-2 targeted therapies. ${ }^{11} A$ prospective study revealed that survival rates of $B C$ patients with $B M$ who were treated with trastuzumab, were increased significantly. ${ }^{6}$ After trastuzumab, HER-2 positive BCpatients have started to use lapatinib, pertuzumab and TDM1. In phase 3 Cleopatra clinical trial, ${ }^{12-14}$ taxane was used with trastuzumab or pertuzumab, and it improved the overall survival (OS) and progression-free survival (PFS) significantly. Moreover, novel trastuzumab deruxtecan and tucatinib agents, which have been shown to have positive results, have started to be used in anti-HER2-based treatments. ${ }^{15,16}$ With this result, taxane use was approved for the firstline treatment of HER-2 positive metastatic $B C .{ }^{12-14}$ In addition, taxane was shown to delay BM. ${ }^{17}$ There is limited data on the prognosis, incidence, treatment, risk factors and complications of $B C$ patients with $B M$ because most clinical trials excluded these patients.

In light of the current literature, the aim of this study was to determine the characteristics and prognosis of brain metastasized HER2-positive BC patients. 


\section{METHODOLOGY}

It was a descriptive study conducted at Ankara Oncology Training and Research Hospital, Turkey between 2000-2019; 3,100 patients were analysed retrospectively and 105 patients met the criteria for inclusion in the study. Age 18 years and over, HER-2 positive BC, and presence of BM were the study inclusion criteria. Patients with secondary malignancies, those with missing data and irregular follow-up were excluded from the study. Age, type of treatment, Eastern Cooperative Oncology Group performance status (ECOG PS) score, BM date, and last contact date of the patients were obtained from the hospital records, retrospectively. Ethics Committee approval was obtained before the study (No. 2020/87).

Statistical analyses were performed using SPSS version 20.0 software (SPSS, Chicago, IL, USA). Qualitative data were given as frequencies and percentages (IQR: 25th percentile-75th percentile). Survival was analysed by Kaplan-Meier method and comparison between groups was performed by the log-rank test. Prognostic factors that affect survival were determined by Cox proportional hazards model multivariate analyses. A pvalue under 0.05 was considered as statistically significant.

\section{RESULTS}

One hundred and five HER-2 positive BC patients whose BM occurred during the course of the disease, were retrospectively examined. The median age of the patients was 48 (IQR 41.5-54.5) years. Forty-one (39\%) patients were hormone receptor (HR) negative, while 61 (58.1\%) patients were HR-positive. HR status of 3 patients could not be found from their records. Nine $(8.6 \%)$ participants had BM at the time of the diagnosis; whereas, $26(24.8 \%)$ patients had metastasis to organs other than the brain at the time of the diagnosis. Overall, 35 (33.3\%) patients had de-novo metastasis (metastatic disease at the time ofdiagnosis).

Patients were given first and second line treatment after metastasis (1-5). As the first line of the treatment, 54 (51.4) patients used taxane and trastuzumab, 19 (18.1\%) patients used capecitabine and lapatinib, 7 (6.7\%) patients used vinorelbineand trastuzumab, 4(3.8\%) patients used pertuzumab, trastuzumab, and taxane, and $3(2.9 \%)$ patients used TDM-1 therapy. All patients were able to get trastuzumab treatment; whereas, 26 (24.8) patients were given TDM-1 treatment and 4 (3.8\%) patients used dual anti-HER-2 treatment with pertuzumab and trastuzumab, at anystage of the treatment.

Ninety-two (87.6\%) of the 105 retrospectively examined patients died. Median OS was found to be 50 months $(95 \% \mathrm{Cl}=$ 39.6-60.4) in all patients. Median OS was 43 months (35.5-50.5) for HR-negative patients, while it was determined to be 59 months (44.5-73.5) in the HR-positive patients ( $p=0.035$, Figure 1a). In addition, median OS was 31 months (25.1-36.9) and 66 months (52.2-79.8) for patients with de-novo metastasis (metastatic disease at the time of diagnosis) and patients with no metastasis at the time of the diagnosis, respectively $(p<0.001$, Figure $1 \mathrm{~b}$ ). The median OS between 25 patients with one BM and
80 patients with multiple BM was found to be 90 months (65.6-114.4) in the first-mentioned group and 45 months (38.4-51.6) in the latter group ( $p=0.003$, Figure $1 c)$. Moreover, median OS was 90 months (62.6-117.4) for patients who underwent surgery for BM whereas it was 47 months (41-53) for patients who did not undergo surgery for $\mathrm{BM}(p=0.034)$. Also, median OS was determined to be 50 months (37.7-62.3) and 47 months (25.5-68.5) for patients who were given cranial radiotherapy and patients who did not receive cranial radiotherapy, respectively $(p=0.885$, Tablel).

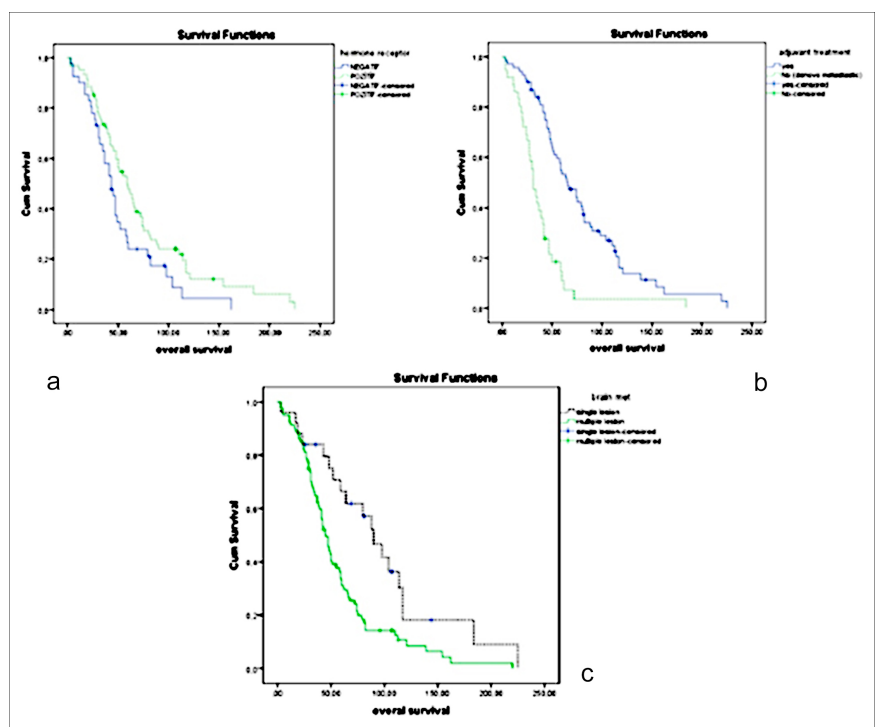

Figure 1: (a) Median overall survival for hormone receptor status positive and negative. (b) Median overall survival for de novo metastasis and nonmetastatis at the diagnosis time. (c) Median overall survival for single and multiple brain metastasis.

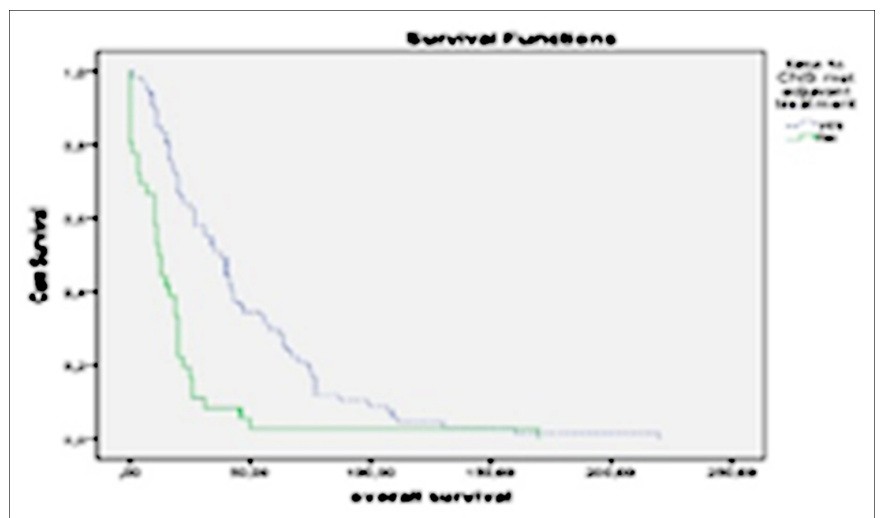

Figure 2: Median time to progression of BM for de novo metastasis and non-metastasis patients.

In all patients, the median time to BM was 22 months (17.02-26.97). Median time to occurence of BM was 15 months (9.2-20.8) for patients with extracranial metastatic disease at the time of diagnosis, while it was 35 months (23.3-46.7) for patients with no metastasis at the time of the diagnosis, $(p=0.001$, Figure 2). The time to brain metastasis in the whole patient population was 19 months (14.2-23.8) in the high grade group and 31 months in the low grade group.(12.1-49.9) $(p=0.06)$. The time to brain metastasis in the receptor positive group was 26 months (16.6-35.4) while it was 20 months in the hormone receptor negative group (15.9-24.1, $p=0.136$ ). Multivariate cox regression anal- 
yses revealed that time to occurence of BM was shorter in patients with high-grade tumors compared to patients with low-grade tumors (HR: $1.64,95 \% \mathrm{Cl}: 1.0-2.7) p=0.046$ ), and in patients with de-novo metastasis compared to patients without de-novo metastasis (HR: 0.44, 95\% Cl: 0.2-0.8, $\mathrm{p}=0.006$ ).

Table I: Trial population median overall survival(OS).

\begin{tabular}{|c|c|c|c|}
\hline & Patients (n) & $\begin{array}{l}\text { Median OS } \\
\text { (months) }\end{array}$ & p-value \\
\hline \multicolumn{4}{|l|}{ Hormon receptor status } \\
\hline Positive & $41(39 \%)$ & 59 & \multirow{3}{*}{0.035} \\
\hline Negative & $61(58.1 \%)$ & 43 & \\
\hline Unknown & $3(2.9 \%)$ & - & \\
\hline \multicolumn{4}{|l|}{ Metastatic type } \\
\hline De-novo metastatic & $35(33.3 \%)$ & 31 & \multirow{3}{*}{$<0.001$} \\
\hline $\begin{array}{l}\text { De-novo brain } \\
\text { metastatic }\end{array}$ & $9(8.6 \%)$ & - & \\
\hline $\begin{array}{l}\text { Non De-novo } \\
\text { metastatic }\end{array}$ & $70(66.7 \%)$ & 66 & \\
\hline \multicolumn{4}{|l|}{ Brain metastatis } \\
\hline Single & $25(23.8 \%)$ & 90 & \multirow{2}{*}{0.003} \\
\hline Multiple & $80(76.2 \%)$ & 45 & \\
\hline \multicolumn{4}{|l|}{ Cranial RT } \\
\hline Recieved & $93(88.6 \%)$ & 50 & \multirow{2}{*}{0.885} \\
\hline Not recieved & $12(11.4 \%)$ & 47 & \\
\hline
\end{tabular}

Table II: Multivariate analysis of the effect of ECOG PS, number of brain metastasis, surgery of brain metastasis.

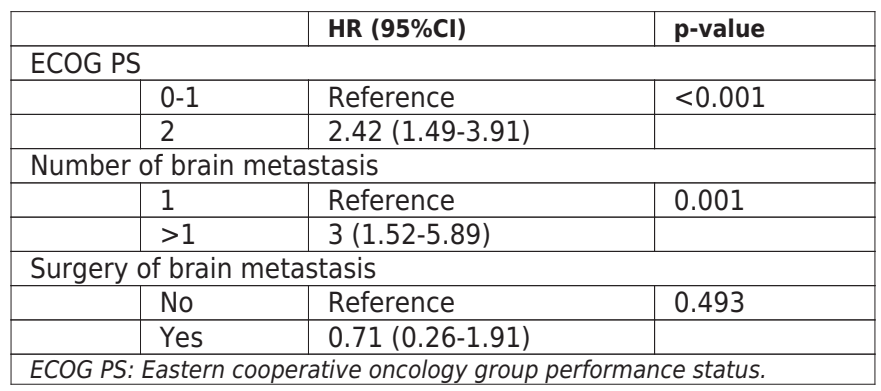

Median OS was determined to be 15 (12.4-17.6) months for all patients with BM after the occurrence of the metastasis. Patients with Eastern Cooperative Oncology Group performance status (ECOG PS) score of $0-1$ at the time of the BM had 19 months (12.3-25.7) median OS, while patients with ECOG PS score of 2 at the time of the BM had 8 months (5.7-10.3) median OS ( $p<0.001)$. Median OS after BM was 32 months (3.9-60.1) and 14 months (11.5-16.5) for patients with one BM and patients with multiple $\mathrm{BM}$, respectively $(p=0.001)$. In addition, median OS after BM was determined to be 64 months (23.9-104.1) for patients who underwent brain surgery and it was found to be 14 months (11.6-16.4) for patients who did not undergo brain surgery $(p=0.010)$. Further, median OS after BM was 31 months (8.6-53.4) for patients with only BM and it was 13 months (11.1-14.9) for patients with metastasis to an organ other than the brain in addition to $B M(p=$ 0.018).

ECOG PS score and the number of BM were accepted as independent predictive factors for median OS during multivariate Cox regression analyses. A worse prognosis was observed in patients with ECOG PS score of 2 compared to patients with better PS and in patients with multiple BM compared to patients with solitary BM (Tablell).

\section{DISCUSSION}

In this study, it was found that tumor grade and de-novo metastasis (extracranial metastasis at the time of diagnosis without brain metastasis) affected the occurrence of BM. On the other hand, it was showed that the number of BM and ECOG PS score were the factors, which affectedOS.

It is known that HER-2 positive or triple negative $B C$ patients have a high rate of $B M .^{3,5-9}$ In addition, tumor grade and $\mathrm{HR}$ status are the risk factors for central nervous system metastasis. ${ }^{6,18}$ In this study, the median OS for all patients was found to be 50 months, for HR-positive patients it was found to be 59 months and for HR-negative patients, it was 43 months. There are different results in the literature regarding $O S$. These results on OS seem to be better compared to most studies in the literature. ${ }^{19}$ This finding might be the result of that $33.3 \%$ of patient population had de-novo metastasis and $8.6 \%$ of patient population had BM at the time of the diagnosis. A study performed by Honda et al. found that the median OS for HR and HER-2 positive patients was 37 months. ${ }^{20}$ Difference between this study and aforementioned study regarding median OS could be due to the fact that there were more $\mathrm{BC}$ patients with multiple BM, median ECOG PS score was worse in their patient population and the number of recruits was smaller in Honda et al. study. ${ }^{20}$ In parallel with the literature, this study revealed that HR-positive patients had a better prognosis compared to HR-negative patients. Besides, different OS lengths between different studies might be the result of the number and size of BM of patients and changing local treatments for metastasis among studies. ${ }^{12-14,17-20}$ Despite the relatively low number of patients, who underwent surgery for metastasis removal in our study, these patients had better OS compared to patients who did not have surgery (median OS were 90 and 47 months, respectively). There was no statistically significant difference between the median OS for patients treated with cranial radiotherapy and patients who did not. This result might due to the low number of patients who did not receive cranial radiotherapy.

Median time to occurence of BM in this study was 22 months. A previous study reported that high tumor grade was an independent predictive factor affecting the time for the occurrence of BM. ${ }^{18}$ It was found the same result, as well. In addition, this study showed that de-novo metastasis was also an independent predictive factor affecting the time for the occurrence of BM. It was also found that HR-negative patients had a worse prognosis compared to HR-positive patients, like the mentioned study.

Previous studies found that median OS after BM varies between 6.4 and 12 months. ${ }^{21-23}$ Median OS after BM in the present study was 15 months. The finding of better median OS after BM might be due to the low number of recruited patients in some previous studies and inclusion of patients with bad prognosis who were given multiple step treatment before BM in some others.

Similar to a previous study, this study found a better median OS for patients with only BM compared to patients with other organ metastasis, in addition to $\mathrm{BM}^{24}{ }^{24}$ The mentioned study reported 
worse median OS compared to our study (median OS for patients with only BM was 13.7 months). This finding might be the result of the recruitment of patients other than HER-2 positive patients in that study. Moreover, this finding supported the claim that anti-HER-2 treatments improve OS for HER-2 positive $B C$ patients. When examined the factors affecting OS in this study, it was found that patients with worse ECOG PS score, multiple BM or inoperable BM had a worse prognosis.

There are several potential limitations to this study. First, the study is a retrospective single-centre study; and it is notideal for early-period patients to have access to anti-HER-2 treatments due to the long inclusion process. Additionally, there is no official approval of pertuzumab due to no health insurance payment in Turkey afteruse TDM-1

\section{CONCLUSION}

This study indicated that tumor grade and de-novo metastasis (extracranial metastasis at the time of diagnosis) are independent predictive factors that may cause the earlier occurrence of $\mathrm{BM}$, and affect mortality in BC patients. After the occurrence of $B M$, lowerECOG PS score and multiple BM at the time of the diagnosis affected median OS as poor prognostic factors.

\section{ETHICALAPPROVAL:}

This study was conducted in compliance with the ethical principles according to the Declaration of Helsinki, and it was approved by the local Institutional Review Board (No. 2020/87).

\section{PATIENTS' CONSENT:}

Since it was designed as a retrospective study, the data were collected from the hospital archive after approval of the Ethics Committee.

\section{CONFLICT OF INTEREST:}

The authors declared no conflict of interest.

\section{AUTHORS' CONTRIBUTION:}

SK, IK, OA, OBSO, UD: Conceived the study design, involved in data collection, performed the statistical analysis, interpreted data, and prepared the manuscript draft.

All the authors critically reviewed the final version of the manuscript and approved it for publication.

\section{REFERENCES}

1. Imamoglu GI, Eren T, Arzu O, Yıldırım N, Karacin C, Baylan B. Is Tamoxifen Use a Factor Affecting Continence in Breast Cancer Patients? Cureus 2019; 11(8):e5417. doi: 10.7759/ cureus.5417.

2. Goldhirsch A, Winer EP, Coates A, Gelber R, Piccart-Gebhart M, Thürlimann $B$, et al. Personalising the treatment of women with early breast cancer: Highlights of the St Gallen international expert consensus on the primary therapy of early breast cancer 2013. Ann Oncol 2013; 24(9):2206-2223. doi: 10.1093/ annonc/mdt303.

3. Kennecke H, Yerushalmi R, Woods R, Cheang MCU, Voduc D, Speers $\mathrm{CH}$, et al. Metastatic behavior of breast cancer subtypes. J Clin Oncol 2010; 28(20):3271-3277. doi: 10.1200/ JCO.2009.25.9820.

4. Martin AM, Cagney DN, Catalano PJ, Warren LE, Bellon JR,
Punglia RS, et al. Brain metastases in newly diagnosed breast cancer: A population-based study. JAMA oncology 2017; 3(8):1069-1077. doi: 10.1001/jamaoncol.2017.0001.

5. Pestalozzi BC, Zahrieh D, Price K, Holmberg S, Lindtner J, Collins J, et al. Identifying breast cancer patients at risk for Central Nervous System (CNS) metastases in trials of the International Breast Cancer Study Group (IBCSG). Ann Oncol 2006; 17(6):935-944. doi: 10.1093/annonc/mdl064.

6. Brufsky AM, Mayer M, Rugo HS, Kaufman PA, Tan-Chiu E, Tripathy $D$, et al. Central nervous system metastases in patients with HER2-positive metastatic breast cancer: incidence, treatment, and survival in patients from registHER. Clin Cancer Res 2011; 17(14):4834-4843. doi: 10.1158/ 1078-0432.CCR-10-2962.

7. Musolino A, Ciccolallo L, Panebianco M, Fontana E, Zanoni D, Bozzetti $C$, et al. Multifactorial central nervous system recurrence susceptibility in patients with HER2-positive breast cancer: Epidemiological and clinical data from a populationbased cancer registry study. Cancer 2011; 117(9):1837-46. doi: 10.1002/cncr.25771.

8. Olson EM, Najita JS, Sohl J, Arnaout A, Burstein HJ, Winer EP, et al. Clinical outcomes and treatment practice patterns of patients with HER2-positive metastatic breast cancer in the post-trastuzumab era. The Breast 2013; 22(4):525-531. doi: 10.1016/j.breast.2012.12.006.

9. Aversa C, Rossi V, Geuna E, Martinello R, Milani A, Redana S, et al. Metastatic breast cancer subtypes and central nervous system metastases. The Breast 2014; 23(5):623-8. doi: 10.1016/j.breast.2014.06.009.

10. Leone JP, Leone BA. Breast cancer brain metastases: The last frontier. Exp Hematol Oncol 2015; 4(1):1-10. doi: 10.1186/ s40164-015-0028-8.

11. Rostami R, Mittal S, Rostami P, Tavassoli F, Jabbari B. Brain metastasis in breast cancer: A comprehensive literature review. J Neurooncol 2016; 127(3):407-414. doi: 10.1007/ s11060-016-2075-3.

12. Baselga J, Cortés J, Kim SB, Im SA, Hegg R, Im YH, et al. Pertuzumab plus trastuzumab plus docetaxel for metastatic breast cancer. N Engl J Med 2012; 366(2):109-119. doi: 10.1056/NEJMoa1113216.

13. Luen SJ, Salgado R, Fox S, Savas P, Eng-Wong J, Clark E, et al. Tumour-infiltrating lymphocytes in advanced HER-2 positive breast cancer treated with pertuzumab or placebo in addition to trastuzumab and docetaxel: A retrospective analysis of the Cleopatra study. Lancet Oncol 2017; 18(1):52-62. doi: 10.1016/S1470-2045(16)30631-3.

14. Swain SM, Baselga J, Kim SB, Ro J, Semiglazov V, Campone M, et al. Pertuzumab, trastuzumab, and docetaxel in HER2positive metastatic breast cancer. N Engl J Med 2015; 372(8): 724-734. doi: 10.1056/NEJMoa1413513.

15. Modi S, Saura C, Yamashita T, Park YH, Kim SB, Tamura K, et al. Trastuzumab deruxtecan in previously treated HER-2 positive breast cancer. N Engl J Med 2020; 382(7):610-21. doi: 10.1056/NEJMoa1914510.

16. Murthy RK, Loi S, Okines A, Paplomata E, Hamilton E, Hurvitz $\mathrm{SA}$, et al. Tucatinib, trastuzumab, and capecitabine for HER-2 positive metastatic breast cancer. N Engl J Med 2020; 382(7):597-609. doi: 10.1056/NEJMoa1914609.

17. Swain S, Baselga J, Miles D, Im YH, Quah C, Lee L, et al. Incidence of central nervous system metastases in patients with HER-2 positive metastatic breast cancer treated with pertuzumab, trastuzumab, and docetaxel: Results from the 
randomised phase III study Cleopatra. Ann Oncol 2014; 25(6):1116-1121. doi: 10.1093/annonc/mdu133.

18. Chow L, Suen D, Ma KK, Kwong A. Identifying risk factors for brain metastasis in breast cancer patients: Implication for a vigorous surveillance program. Asian J Surg 2015; 38(4):220-223. doi: 10.1016/j.asjsur.2015.03.003.

19. Andersson $M$, Lidbrink $E$, Bjerre $K$, Wist $E$, Enevoldsen $K$, Jensen $A B$, et al. Phase III randomized study comparing docetaxel plus trastuzumab with vinorelbine plus trastuzumab as first-line therapy of metastatic or locally advanced human epidermal growth factor receptor 2-positive breast cancer: The Hernata study. J Clin Oncol 2011; 29(3):264-271. doi: 10.1200/JCO. 2010.30.8213.

20. Honda $Y$, Aruga T, Yamashita T, Miyamoto H, Horiguchi K, Kitagawa $\mathrm{D}$, et al. Prolonged survival after diagnosis of brain metastasis from breast cancer: Contributing factors and treatment implications. Jpn J Clin Oncol 2015; 45(8):713-718. doi: 10.1093/jjco/hyv067.

21. Lin NU, Diéras V, Paul D, Lossignol D, Christodoulou C,
Stemmler $\mathrm{H}-\mathrm{J}$, et al. Multicenter phase II study of lapatinib in patients with brain metastases from HER-2 positive breast cancer. Clin Cancer Res 2009; 15(4):1452-1459. doi: 10.1158/1078-0432.CCR-08-1080.

22. Bachelot T, Romieu G, Campone M, Diéras V, Cropet C, Dalenc $\mathrm{F}$, et al. Lapatinib plus capecitabine in patients with previously untreated brain metastases from HER-2 positive metastatic breast cancer (Landscape): A single-group phase 2 study. Lancet Oncol 2013; 14(1):64-71. doi: 10.1016/S1470- 2045 (12)70432-1.

23. Freedman RA, Gelman RS, Wefel JS, Melisko ME, Hess KR, Connolly RM, et al. Translational Breast Cancer Research Consortium (TBCRC) 022: A phase II trial of neratinib for patients with human epidermal growth factor receptor 2-positive breast cancer and brainmetastases. J Clin Oncol 2016; 34(9):945. doi: 10.1200/JCO.2015.63.0343.

24. Arslan UY, Oksuzoglu B, Aksoy S, Harputluoglu H, Turker I, Ozisik $Y$, et al. Breast cancer subtypes and outcomes of central nervous system metastases. Breast 2011; 20(6):562-7. doi.org/10.1016/j.breast.2011.07.017. 